\title{
Correction to: Improving accuracy of opening-wedge osteotomies of distal radius using a patient-specific ramp-guide technique
}

Simon Roner ${ }^{1 *}$, Fabio Carrillo ${ }^{1}$, Lazaros Vlachopoulos ${ }^{2}$, Andreas Schweizer ${ }^{2}$, Ladislav Nagy ${ }^{2}$ and Philipp Fuernstahl ${ }^{1}$

\section{Correction}

Following publication of the original article [1], the author pointed out that the references were numbered incorrectly. This error was introduced during the production process. The original article has been corrected.

\section{Author details}

${ }^{1}$ Computer Assisted Research and Development Group, Balgrist University Hospital, University of Zurich, Forchstrasse 340, 8008 Zurich, Switzerland.

${ }^{2}$ Department of Orthopaedics, Balgrist University Hospital, University of

Zurich, Zurich, Switzerland.

Published online: 19 November 2018

\section{Reference}

1. Roner S, et al. Improving accuracy of opening-wedge osteotomies of distal radius using a patient-specific ramp-guide technique. BMC Musculoskelet Disord. 2018;19:374. https://doi.org/10.1186/s12891-018-2279-0.

\footnotetext{
* Correspondence: simon.roner@balgrist.ch

${ }^{1}$ Computer Assisted Research and Development Group, Balgrist University

Hospital, University of Zurich, Forchstrasse 340, 8008 Zurich, Switzerland
} 